\title{
Improved optical limiting performance of laser-ablation-generated metal nanoparticles due to silica-microsphere-induced local field enhancement
}

\author{
Zheren $\mathrm{Du}^{1}$, Lianwei Chen ${ }^{2}$, Tsung-Sheng $\mathrm{Kao}^{3}$, Mengxue $\mathrm{Wu}^{1}$ and Minghui Hong ${ }^{* 1}$
}

\author{
Full Research Paper \\ Address: \\ ${ }^{1}$ Department of Electrical and Computer Engineering, National \\ University of Singapore, ${ }^{2}$ Engineering Science Program, National \\ University of Singapore and ${ }^{3}$ Department of Photonics and Institute of \\ Electro-Optical Engineering, National Chiao Tung University, $1001 \mathrm{Ta}$ \\ hsueh Rd., Hsinchu 30010, Taiwan \\ Email: \\ Minghui Hong* - elehmh@nus.edu.sg \\ * Corresponding author \\ Keywords: \\ laser ablation; local field enhancement; microspheres; nanoparticles; \\ optical limiting
}

Beilstein J. Nanotechnol. 2015, 6, 1199-1204. doi:10.3762/bjnano.6.122

Received: 12 December 2014

Accepted: 01 May 2015

Published: 22 May 2015

Associate Editor: N. Motta

(c) 2015 Du et al; licensee Beilstein-Institut. License and terms: see end of document.

\begin{abstract}
For practical application, optical limiting materials must exhibit a fast response and a low threshold in order to be used for the protection of the human eye and electro-optical sensors against intense light. Many nanomaterials have been found to exhibit optical limiting properties. Laser ablation offers the possibility of fabricating nanoparticles from a wide range of target materials. For practical use of these materials, their optical limiting performance, including optical limiting threshold and the ability to efficiently attenuate high intensity light, needs to be improved. In this paper, we fabricate nanoparticles of different metals by laser ablation in liquid. We study the optical nonlinear properties of the laser-generated nanoparticle dispersion. Silica microspheres are used to enhance the optical limiting performance of the nanoparticle dispersion. The change in the optical nonlinear properties of the laser-generated nanoparticle dispersion caused by silica microspheres is studied. It is found that the incident laser beam is locally focused by the microspheres, leading to an increased optical nonlinearity of the nanoparticle dispersion.
\end{abstract}

\section{Introduction}

Laser ablation in liquid (LAL) is a versatile technique to fabricate nanoparticles. Conventional synthesis of nanoparticles by chemical reactions is usually complicated and uses toxic materials. The chemically synthesized nanoparticles are easily contaminated by chemical precursors, additives or byproducts. LAL has many advantages, such as the simple, safe, and high purity properties of the generated nanoparticles, the variety of the target materials capable of being produced, and the in situ dispersion of the nanoparticles. The properties of the lasergenerated nanoparticles, such as size, size distribution, composition, and structure for different target materials can be flexibly controlled by the laser parameters (e.g., wavelength, repetition rate, pulse duration, and laser fluence) and the properties of the environment (e.g., surfactant concentration, $\mathrm{pH}$ value, or size 
and length of ligands) [1-6]. Laser ablation is an explosive material removal process using strong pulsed-laser irradiation. The LAL process causes the ejection of nanoclusters into the environment to form a colloidal nanoparticle dispersion [7-9]. The nanoparticles have wide application in physics, chemistry, biology and engineering. One of the important applications is that the nanoparticles can be used as optical limiting materials for the protection of the human eye and in optical devices against high intensity light irradiation.

A material exhibiting the optical limiting effect has a decreased optical transmittance with increasing incident light intensity. A good optical limiter should have high transparency at low light irradiance, while it efficiently attenuates high incident light irradiance. Nonlinear optical material interactions, such as nonlinear absorption, refraction and scattering [10-12], are the main optical processes which contribute to optical limiting. Various materials have been found to exhibit optical limiting properties, such as fullerenes, metallophthalocyanines, carbon black suspension and nanoscale metallic materials [13-15].

For the practical use of the optical limiting materials, it is important to improve their optical limiting performance, including a lower optical limiting threshold and the ability to efficiently attenuate high intensity light. In this paper, we fabricate nanoparticles of different materials by LAL. We investigate the optical nonlinear properties of the laser-ablation-gener- ated nanoparticle dispersion. A novel method is proposed to improve the optical nonlinear properties of the dispersion by coupling with silica microspheres. It is found that the incident laser beam is locally focused by the microspheres, leading to an improved optical nonlinearity of the nanoparticle dispersion. The optical limiting threshold of the optical limiting material is significantly lowered due to the enhanced nonlinear refraction.

\section{Experimental Materials}

The nanoparticle dispersions were fabricated by the LAL technique as schematically shown in Figure 1a. A solid target material was placed at the bottom of a beaker and immersed in $10 \mathrm{~mL}$ of deionized (DI) water. A fiber laser with a wavelength of $1064 \mathrm{~nm}$, a pulse duration of $\approx 10 \mathrm{~ns}$ and a repetition rate of $100 \mathrm{kHz}$, was focused onto the surface of the target material with a spot size of $\approx 30 \mu \mathrm{m}$. The applied laser fluence was $12.5 \mathrm{~J} / \mathrm{cm}^{2}$. The laser beam was programmed to scan over a $1 \times 1 \mathrm{~cm}^{2}$ area 900 times. A square scanning pattern was chosen and the scanning speed was set at $1 \mathrm{~mm} / \mathrm{s}$. The entire process took about $5 \mathrm{~min}$. The laser-generated nanoparticles were dispersed into the DI water. The dispersion was collected and sonicated for $30 \mathrm{~min}$ to ensure the uniform distribution of the nanoparticles in the DI water. The target materials included gold and silver of high purity. Silica microspheres (purchased from Thermo Scientific) with a diameter of $1 \mu \mathrm{m}$ were used in this study. The number of microspheres per unit weight was
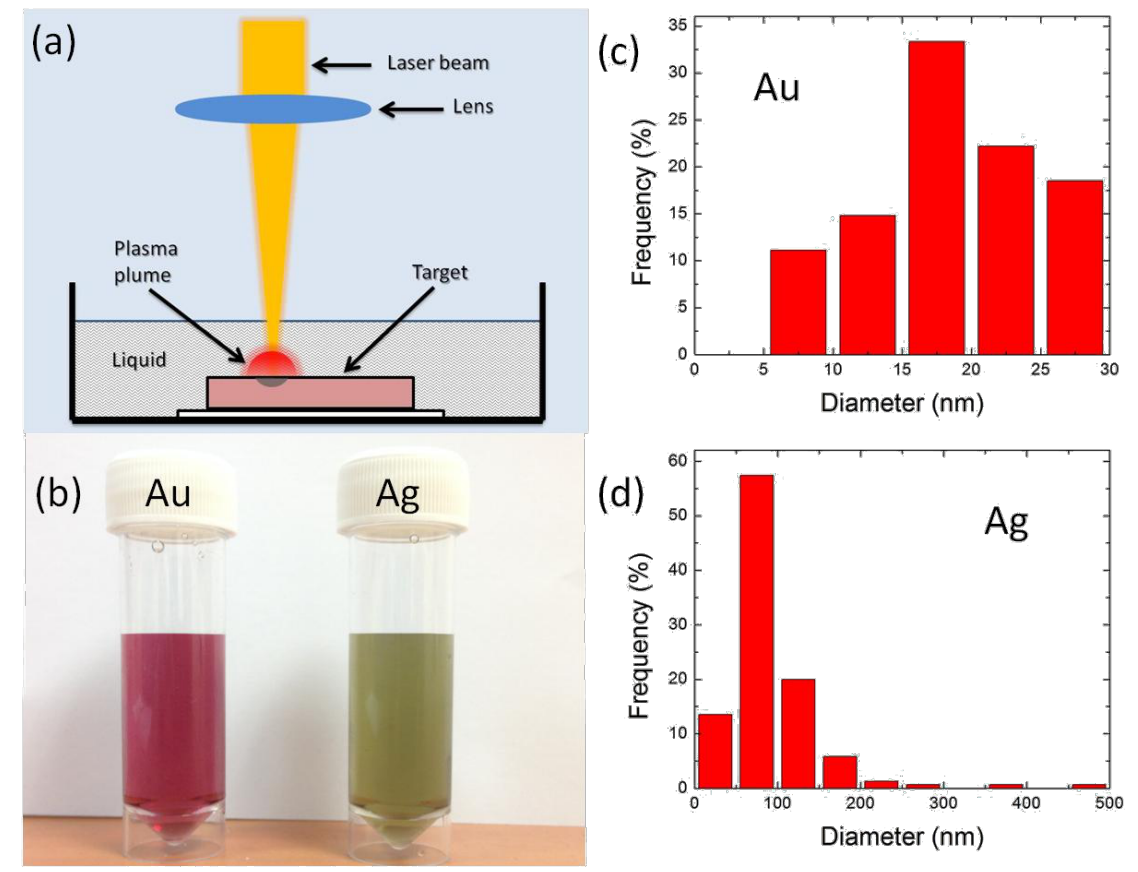

Figure 1: (a) Schematic of the LAL experimental setup. (b) Photograph of laser-generated gold and silver nanoparticle dispersions by the LAL technique. Size distributions of (c) gold and (d) silver nanoparticles estimated from SEM images. 
calculated to be $9 \times 10^{10} \mathrm{~g}^{-1} .0 .2 \mathrm{mg}$ of microspheres was uniformly dispersed in a $3 \mathrm{~mL}$ nanoparticle dispersion to form a hybrid material dispersion.

\section{Measurement}

The laser-generated nanoparticles were characterized by scanning electron microscopy (SEM). The size distribution of the nanoparticles was estimated by analyzing the SEM images. The fiber laser for LAL was also used for the optical limiting measurement. The nanoparticle and hybrid material dispersions were placed in a glass cuvette with a light path of $1 \mathrm{~mm}$ for the optical limiting measurement. The incident and transmitted laser powers were measured.

\section{Results and Discussion}

The nanoparticle dispersions of different materials were fabricated by LAL as shown in Figure 1a. LAL is an explosive material removal process that can be used for the synthesis of nanoparticles. The interaction between ns laser pulses and a solid target in a liquid environment typically involves plasma formation, expansion and shock wave generation [16,17]. A high density of energetic species is generated in the plasma plume during the ablation process. The energetic species includes atoms, molecules, ions, clusters, and ultrafine particulates. The strong interaction among these energetic species and liquid molecules promotes nucleation and aggregation, leading to the formation of the nanoparticles [1].

Figure $1 \mathrm{~b}$ shows a photograph of the laser-generated gold and silver nanoparticle dispersions fabricated by the LAL technique. Droplets of the nanoparticle colloidal solution were placed on a polished silicon substrate. These droplets were dried and the nanoparticles on the silicon substrate were characterized by SEM. The particle size and size distribution of the gold and silver nanoparticles are summarized in Figure 1c,d. This shows that the gold nanoparticle diameter distribution ranges from 5 to $30 \mathrm{~nm}$ with a maximum at $15 \mathrm{~nm}$. The effect of clustering of the nanoparticles is observed upon drying of the colloidal solution. The silver nanoparticle diameters range from 20 to $500 \mathrm{~nm}$ with maximum at $80 \mathrm{~nm}$. It can be seen that a few Ag nanoparticles have a relatively larger diameter in the range of a few hundred nanometers. Comparing these two types of nanoparticles, the Au nanoparticles have a smaller average particle size and better particle uniformity. Since the laser parameters used for the generation of the nanoparticles for both $\mathrm{Au}$ and $\mathrm{Ag}$ are the same, the difference in the size distribution of the generated nanoparticles is not only due to the laser parameters but also several other factors. One factor is the different thickness of the target sample, which leads to the slightly different DI water layer thickness above the target sample surface. The laser ablation rate can be enhanced by the confinement of the water [18]
For the Au target, the water layer thickness was $\approx 1 \mathrm{~mm}$, while for the Ag target, the water layer thickness was $\approx 5 \mathrm{~mm}$. For the $\mathrm{Au}$ target, the plasma generated during laser ablation is effectively confined by the $1 \mathrm{~mm}$ thick water film, leading to an increased plasma pressure and temperature. Thus, a large amount of energetic species are ejected during the laser ablation. The generated nanoparticles remain in the incident laser light path due to the limited mobility of the nanoparticles in water and the limited volume of the beaker. These nanoparticles can absorb the subsequent laser pulse energy. The size of the nanoparticles that absorb the incident laser light decreases because of the laser-induced fragmentation $[19,20]$. For the Ag target, having a much thicker water layer, the laser ablation rate is reduced because of the absorption of the laser energy by water. In addition, the laser-induced fragmentation is also reduced due to fewer ablated species and less absorbed laser energy. Hence, the size of the laser-generated Ag nanoparticles is larger. Au and Ag have different physical properties, such as the absorption spectrum of the laser light, melting point, boiling point and thermal conductivity, which all can contribute to the size difference.

The laser-generated metallic nanoparticles are promising optical limiting materials. Several mechanisms, as discussed in the Introduction, could be responsible for the optical limiting behavior of these materials. Under the illumination of ns laser pulses at $1064 \mathrm{~nm}$, the optical limiting effect of Au nanoparticles was believed to be due to nonlinear scattering. Polavarapu et al. [21] have shown that nonlinear absorption was not significant for the gold nanoparticles in water. At low laser fluence, the nanoparticle dispersion is almost transparent to the laser light and thus the laser light exhibits a linear transmission through the dispersion as shown in Figure 2a. The optical limiting effect of the nanoparticles appears upon formation of microbubbles. The bubble formation occurs at the solvent-nanoparticle interface. The absorbed photon energy by the nanoparticle is dissipated as heat to the surrounding solvent, leading to solvent vaporization and bubble formation. These bubbles act as scattering centers, which induces the optical limiting effect [22]. Typically, the contribution from nonlinear scattering becomes dominant at high laser fluence as illustrated in Figure 2b.

To verify the optical limiting performance of the laser-generated nanoparticles, the nonlinear transmission of these nanoparticles was investigated using a fiber laser operated at a wavelength of $1064 \mathrm{~nm}$ and a pulse duration of $\approx 10 \mathrm{~ns}$. Figure 3 shows the optical limiting performance and the nonlinear transmission results. Both materials ( $\mathrm{Au}$ and $\mathrm{Ag}$ ) exhibited a significant optical limiting effect. The threshold for the optical limiting effect is defined as the laser pulse fluence at which the 


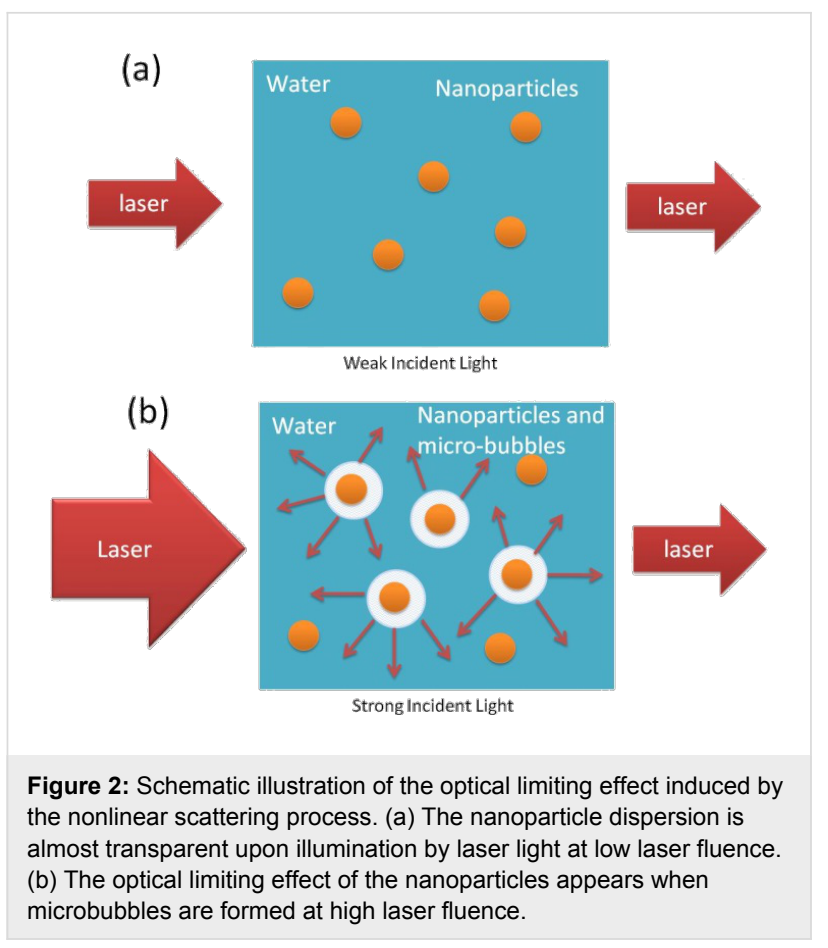

bubble starts to form, causing significant nonlinear scattering and fluctuation in the output power measurement, which is due to the movement of the bubbles. Figure 3a shows the optical limiting response for the gold nanoparticle and the gold/silica nanocomposite dispersions. For the gold nanoparticle dispersion, the optical limiting threshold is about $6.2 \mathrm{~J} / \mathrm{cm}^{2}$. For the gold/silica nanocomposite dispersion, the optical limiting effect threshold is about $1.6 \mathrm{~J} / \mathrm{cm}^{2}$. Hence, by adding the silica microspheres, the optical limiting threshold is lowered by $4.6 \mathrm{~J} / \mathrm{cm}^{2}$. Figure $3 \mathrm{~b}$ shows the normalized nonlinear transmittance of the samples (gold nanoparticles and gold/silica nanocomposites) upon excitation with ns laser pulses at $1064 \mathrm{~nm}$. The input laser fluence was varied from 0.3 to $10.6 \mathrm{~J} / \mathrm{cm}^{2}$. As the input fluence is low, the transmission does not change much with input fluence. When the input fluence is further increased, the transmission of all samples starts to decrease. It is clear that the transmittance reduces much more rapidly for the gold/silica nanocomposites than for the pure gold nanoparticle dispersion. Figure 3c,d shows the optical limiting response and normalized transmittance curves of silver nanoparticles and silver/silica nanocomposites. By adding the silica
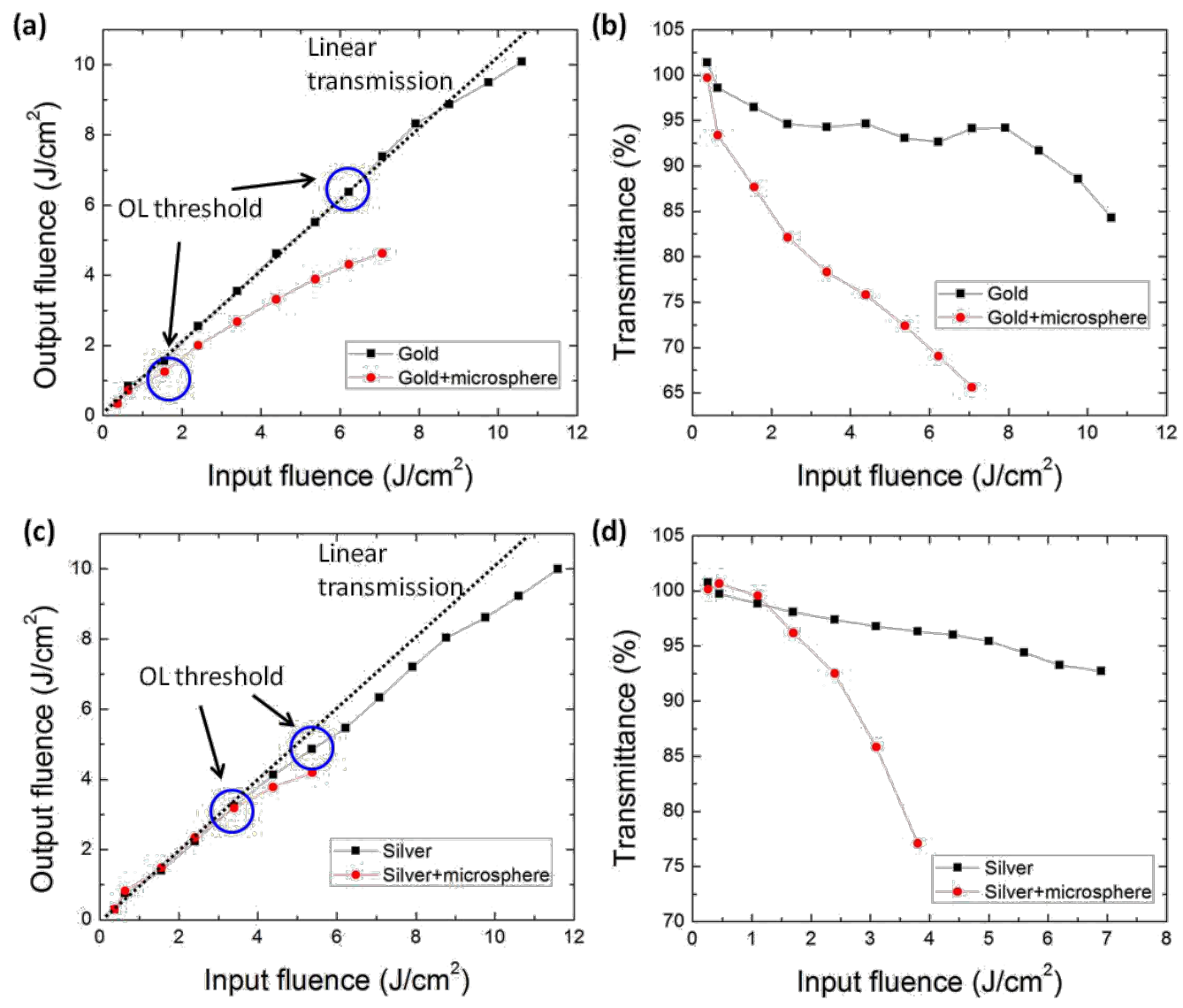

Figure 3: Optical limiting (OL) response and normalized transmittance curves of $(a, b)$ gold nanoparticles and gold/silica nanocomposites and (c,d) silver nanoparticles and silver/silica nanocomposites. 
microspheres, the optical limiting threshold is lowered from 5.4 to $3.4 \mathrm{~J} / \mathrm{cm}^{2}$. The transmittance curves in Figure $3 \mathrm{~d}$ also show that the optical limiting property of the silver nanoparticles can be improved by addition of silica microspheres. From these results, it can be demonstrated that the optical limiting threshold can be significantly lowered with the addition of silica microspheres.

Silica microspheres are known to induce an enhanced local field used for various applications [23,24]. Figure 4 a provides a SEM image of the silica microspheres. It shows that the microspheres have uniform shape and size of $1 \mu \mathrm{m}$. Figure $4 \mathrm{~b}$ shows the simulation result using the finite-difference time-domain (FDTD) method with the Lumerical software for predicting the enhancement of the optical nonlinearity by the microspheres. The refractive index of the environment (water) was set at 1.33, while the refractive index of the silica microsphere was set at 1.51. It is observed that the light energy in the area near the silica microsphere is enhanced due to its focusing effect. The enhancement of the light energy is $\approx 1.4$ times higher than the incident light at the center of the enhanced area. The enhancement of the optical nonlinearity is attributed to the local field effect induced by the light focusing of the silica microspheres. We have also simulated the light energy enhancement for different sizes of microspheres, as shown in Table 1. It is found that larger sized microspheres induce a stronger local field enhancement. In our experiment, we found that large sized $(>1 \mu \mathrm{m})$ microspheres were not stable in the nanoparticle dispersion. The microspheres settle to the bottom of the cuvette after tens of seconds after introducing them in the dispersion. Since the local field enhancement can be increased by larger sized microspheres, high viscosity liquids need to be used instead of DI water to improve the stability of the large sizes microspheres in the dispersion. This will be investigated in future research.

\begin{tabular}{|c|c|}
\hline Microsphere size $(\mu \mathrm{m})$ & Field enhancement factor \\
\hline 0.5 & 1.2 \\
\hline 1 & 1.4 \\
\hline 1.5 & 2.2 \\
\hline 2 & 2.8 \\
\hline 5 & 5.9 \\
\hline
\end{tabular}

The introduction of silica microspheres in a metallic nanoparticle dispersion concentrate the light energy into a smaller spot. Such a process causes strong light scattering or absorption, and thus lowers the optical limiting system's energy threshold. The concentration of light by the transparent microspheres is not limited to a specific wavelength, but rather over a broadband

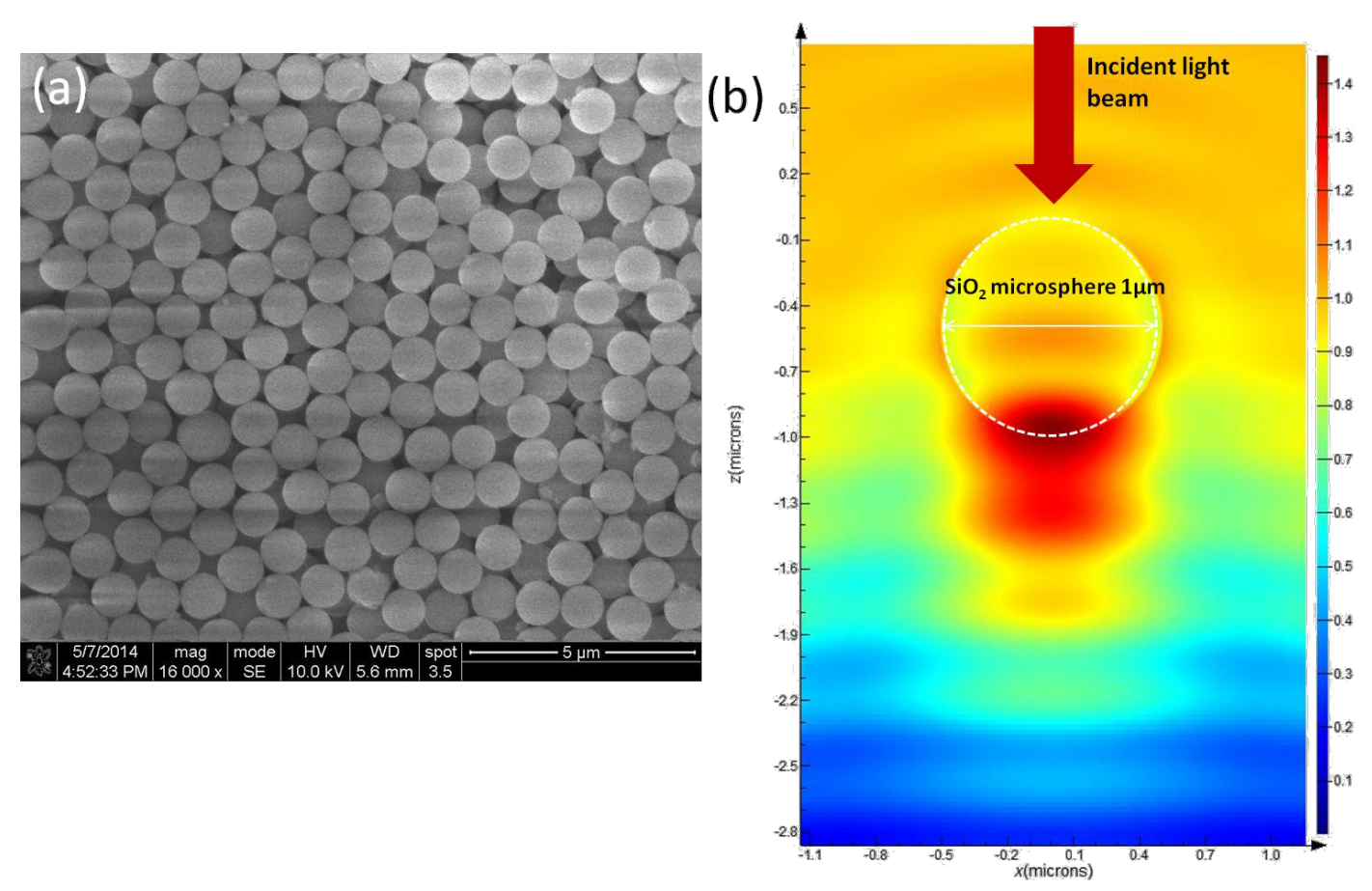

Figure 4: (a) SEM image of silica microspheres and (b) FDTD simulation result for a silica microsphere with a diameter of $1 \mu \mathrm{m}$ under $1064 \mathrm{~nm}$ laser irradiation. 
spectral range, making this hybrid optical limiter more compatible for practical applications. Since the microspheres are transparent in the wavelength range of interest, there will be no visual loss to limit the functionalities. Our optical limiting results have shown that silica microspheres are a promising material to enhance the optical limiting effect. In addition, metallic nanoparticles exhibit localized surface plasmon resonance (LSPR), which is another possible way for local field enhancement to influence the light absorption and scattering [25].

\section{Conclusion}

In this paper, we have studied that LAL is a promising technique to generate nanoparticles for various target materials. These nanoparticles exhibit a strong optical limiting effect. It was found that the optical limiting effect of the nanoparticles can be significantly enhanced by adding silica microspheres. The enhanced optical limiting behavior of the hybrid material is attributed to the interaction between the laser-generated nanoparticles and the silica microspheres, when the metal nanoparticles enter the focal volume of the microspheres. The silica microspheres focus the laser light energy onto the metal nanoparticles, which in turn enhances the optical limiting effect of these nanoparticles.

\section{Acknowledgements}

The authors are grateful for financial support from the Temasek Defence Systems Institute (TDSI) under the project No. R-263000-687-592 TDSI/11-013/1A.

\section{References}

1. Hong, M.; Chen, G.; Chong, T. C. Nanomaterials: Laser-Based Processing in Liquid Media. Nanomaterials: Processing and Characterization with Lasers; Wiley-VCH: Weinheim, Germany, 2012; pp 317-494.

2. Sylvestre, J.-P.; Kabashin, A. V.; Sacher, E.; Meunier, M.; Luong, J. H. T. J. Am. Chem. Soc. 2004, 126, 7176-7177. doi:10.1021/ja048678s

3. Mafuné, F.; Kohno, J.-y.; Takeda, Y.; Kondow, T.; Sawabe, H. J. Phys. Chem. B 2000, 104, 9111-9117. doi:10.1021/jp001336y

4. Schwenke, A.; Wagener, P.; Nolte, S.; Barcikowski, S. Appl. Phys. A 2011, 104, 77-82. doi:10.1007/s00339-011-6398-9

5. Essaidi, A.; Chakif, M.; Schöps, B.; Aumman, A.; Xiao, S.; Esen, C.; Ostendorf, A. J. Laser Micro/Nanoeng. 2013, 8, 131-136.

6. Mafuné, F.; Kohno, J.-y.; Takeda, Y.; Kondow, T.; Sawabe, H. J. Phys. Chem. B 2001, 105, 5114-5120. doi:10.1021/jp0037091

7. Neddersen, J.; Chumanov, G.; Cotton, T. M. Appl. Spectrosc. 1993, 47, 1959-1964. doi:10.1366/0003702934066460

8. Fojtik, A.; Giersig, M.; Henglein, A. Ber. Bunsen-Ges. Phys. Chem. 1993, 97, 1493-1496. doi:10.1002/bbpc.19930971112

9. Chen, Y.-H.; Yeh, C.-S. Colloids Surf., A 2002, 197, 133-139. doi:10.1016/S0927-7757(01)00854-8

10. Tutt, L. W.; Boggess, T. F. Prog. Quantum Electron. 1993, 17, 299-338. doi:10.1016/0079-6727(93)90004-S
11. Chen, P.; Wu, X.; Sun, X.; Lin, J.; Ji, W.; Tan, K. L. Phys. Rev. Lett. 1999, 82, 2548-2551. doi:10.1103/PhysRevLett.82.2548

12. He, G. S.; Tan, L.-S.; Zheng, Q.; Prasad, P. N. Chem. Rev. 2008, 108, 1245-1330. doi:10.1021/cr050054x

13. Shirk, J. S.; Pong, R. G. S.; Flom, S. R.; Heckmann, H.; Hanack, M. J. Phys. Chem. A 2000, 104, 1438-1449. doi:10.1021/jp993254j

14. Sun, Y.-P.; Riggs, J. E. Int. Rev. Phys. Chem. 1999, 18, 43-90. doi:10.1080/014423599230008

15. Mansour, K.; Soileau, M. J.; Van Stryland, E. W. J. Opt. Soc. Am. B 1992, 9, 1100-1109. doi:10.1364/josab.9.001100

16. Lu, Y. F.; Hong, M. H.; Chua, S. J.; Teo, B. S.; Low, T. S. J. Appl. Phys. 1996, 79, 2186-2191. doi:10.1063/1.361182

17. Zhu, S.; Lu, Y. F.; Hong, M. H.; Chen, X. Y. J. Appl. Phys. 2001, 89, 2400-2403. doi:10.1063/1.1342200

18. Zhu, S.; Lu, Y. F.; Hong, M. H. Appl. Phys. Lett. 2001, 79, 1396-1398. doi:10.1063/1.1400086

19. Kamat, P. V.; Flumiani, M.; Hartland, G. V. J. Phys. Chem. B 1998 , 102, 3123-3128. doi:10.1021/jp980009b

20. Link, S.; Burda, C.; Mohamed, M. B.; Nikoobakht, B.; El-Sayed, M. A. J. Phys. Chem. A 1999, 103, 1165-1170. doi:10.1021/jp983141k

21. Polavarapu, L.; Venkatram, N.; Ji, W.; Xu, Q.-H. ACS Appl. Mater. Interfaces 2009, 1, 2298-2303. doi:10.1021/am900442u

22. Francois, L.; Mostafavi, M.; Belloni, J.; Delaire, J. A. Phys. Chem. Chem. Phys. 2001, 3, 4965-4971. doi:10.1039/b106690k

23. Wang, Z.; Guo, W.; Li, L.; Luk'yanchuk, B.; Khan, A.; Liu, Z.; Chen, Z.; Hong, M. Nat. Commun. 2011, 2, No. 218. doi:10.1038/ncomms1211

24. Wang, Z. B.; Hong, M. H.; Luk'yanchuk, B. S.; Lin, Y.; Wang, Q. F.; Chong, T. C. J. Appl. Phys. 2004, 96, 6845-6850. doi:10.1063/1.1786652

25. Yang, J.; Luo, F.; Kao, T. S.; Li, X.; Ho, G. W.; Teng, J.; Luo, X.; Hong, M. Light: Sci. Appl. 2014, 3, e185. doi:10.1038/lsa.2014.66

\section{License and Terms}

This is an Open Access article under the terms of the Creative Commons Attribution License (http://creativecommons.org/licenses/by/2.0), which permits unrestricted use, distribution, and reproduction in any medium, provided the original work is properly cited.

The license is subject to the Beilstein Journal of Nanotechnology terms and conditions: (http://www.beilstein-journals.org/bjnano)

The definitive version of this article is the electronic one which can be found at: $\underline{\text { doi:10.3762/bjnano.6.122 }}$ 